\title{
A human macrophage - hepatocyte co-culture model for comparative studies of infection and replication of Francisella tularensis LVS strain and subspecies holarctica and mediasiatica
}

\author{
Knut Rennert ${ }^{1}$, Peter Otto ${ }^{2}$, Harald Funke 3 , Otmar Huber ${ }^{1,4}$, Herbert Tomaso ${ }^{2}$ and Alexander S. Mosig ${ }^{1,4^{*}}$
}

\begin{abstract}
Background: Francisella tularensis, a gram-negative bacterium replicates intracellularly within macrophages and efficiently evades the innate immune response. It is able to infect and replicate within Kupffer cells, specialized tissue macrophages of the liver, and to modulate the immune response upon infection to its own advantage. Studies on Francisella tularensis liver infection were mostly performed in animal models and difficult to extrapolate to the human situation, since human infections and clinical observations are rare.

Results: Using a human co-culture model of macrophages and hepatocytes we investigated the course of infection of three Francisella tularensis strains (subspecies holarctica - wildtype and live vaccine strain, and mediasiatica wildtype) and analyzed the immune response triggered upon infection. We observed that hepatocytes support the intracellular replication of Franciscella species in macrophages accompanied by a specific immune response inducing TNFa, IL-1 $\beta, I L-6$ and fractalkine (CX3CL1) secretion and the induction of apoptosis.

Conclusions: We could demonstrate that this human macrophage / hepatocyte co-culture model reflects strain-specific virulence of Francisella tularensis. We developed a suitable tool for more detailed in vitro studies on the immune response upon liver cell infection by F. tularensis.
\end{abstract}

Keywords: Francisella tularensis, Human macrophage, Hepatocyte, Co-culture, Liver

\section{Background}

Francisella tularensis (F. tularensis) is a gram-negative bacterium that causes the zoonosis tularemia [1]. Due to its high infectivity it is considered as a class A bioweapon agent [2]. F. tularensis primarily infects and persists in macrophages and is thus used as model bacterium to study adopted strategies to evade primary immune detection [3]. As human infections with $F$. tularensis and related human clinical trials are rare, most of the data available on mechanisms of $F$. tularensis replication are derived from mouse models [4]. However,

\footnotetext{
* Correspondence: alexander.mosig@med.uni-jena.de

${ }^{1}$ Institute of Biochemistry II, Jena University Hospital, 07743 Jena, Germany

${ }^{4}$ Center for Sepsis Control and Care, Jena University Hospital, Jena 07747,

Germany

Full list of author information is available at the end of the article
}

due to interspecies differences in the immune response and opposing evolutionary strategies for resistance vs. tolerance or resilience to infection $[5,6]$ it remains difficult to extrapolate results obtained in animal models to the human situation [7].

Currently, four subspecies (ssp.) of F. tularensis are generally accepted: ssp. tularensis, holarctica, mediasiatica, and novicida. F. tularensis ssp. tularensis is highly virulent in hares and the cause of Type A tularemia, whereas F. tularensis ssp. holarctica is less virulent and causes Type B tularemia; F. tularensis subsp. mediasiatica is a rare pathogen with unique biochemical characteristics that has only been isolated in Kazakhstan and Turkmenistan in Central Asia and exhibits virulence in hares similar to Type B organisms [8]. Since the 
infectious dose of $F$. tularensis wild type strains is very low, the attenuated type B live vaccine strain (LVS) thus has often been used as surrogate for the virulent strains. The LVS strain is able to induce diseases in mice similar to those seen in humans, but possesses practically no risk for laboratory personnel [9-11]. Both, virulent and attenuated strains of $F$. tularensis, survive macrophage phagocytosis. They escape into the cytoplasm by preventing acidification and maturation of the phagosome [12-14].

Kupffer cells represent specialized tissue macrophages within the liver [15] and account for $80-90 \%$ of the total macrophage pool of the body [16]. In response to F. tularensis infection, several morphological alterations of the liver tissue have been observed [17]. In this context, it has been supposed that hepatocytes as well as dendritic cells may support the intracellular replication of $F$. tularensis without undergoing pyroptosis or apoptosis. Moreover, F. tularensis is assumed to delay induction of cell death in host cells to its own advantage until exit from its intracellular environment [2].

So far, there is only little knowledge about the infection and replication cycles of $F$. tularensis within human liver tissue. To investigate these processes in vitro, we co-cultured primary human monocyte-derived macrophages with the recently developed hepatocyte cell line HepaRG, which differentiates into cells with a hepatocyte phenotype and into cells exhibiting a biliary epithelial cell phenotype [18]. In contrast to other hepatic cells lines, i.e. HepG2 or Hep2/C3A, HepaRG cells remain functionally stable during prolonged culture, selforganize with functional bile canaliculi-like structures and respond to inflammatory cytokines [18]. Using this human macrophage / hepatocyte co-culture approach we characterized the infection and replication of $F$. tularensis ssp. holarctica, spp. mediasiatica and the attenuated LVS strain.

\section{Results}

To characterize the impact of hepatocytes on the intracellular replication rate of $F$. tularensis in mono-cell cultures of macrophages or hepatocytes as well as in macrophage/hepatocyte co-cultures, cells were infected with F. tularensis and cultured up to $72 \mathrm{~h}$. The F. tularensis replication rate was measured by flow cytometry using an anti- F. tularensis lipopolysaccharide (LPS) antibody to detect intracellular bacteria. Macrophages were discriminated from hepatocytes in the flow cytometric analyses by combined gating of FSC/SSC scattering and fluorescence measurement of FITC-labeled antibody directed against the leukocyte marker protein CD45. F. tularensis ssp. holarctica and LVS strain were reliably detected in macrophages and hepatocytes. Although it has been concluded from previous studies that all three F. tularensis ssp. possess a common LPS lipid A structure [19], we were unable to detect intracellular LPS after infection with $F$. tularensis ssp. mediasiatica. However, presence and replication of intracellular viable bacteria in the hepatocyte / macrophages co-culture of all three $F$. tularensis strains were confirmed by colony forming unit (CFU) assays from lysates of infected cells (Fig. 1, Additional file 1: Figure S1).

Replication of $F$. tularensis LVS strain and $F$. tularensis $s s p$. holarctica was confirmed in monocultures of macrophages and in hepatocytes (Fig. 2a and b, left part) as well as in the co-culture of both cell types (Fig. 2a and $\mathrm{b}$, right part). Interestingly, the highest LPS content was detected in co-cultures with the lowest macrophage

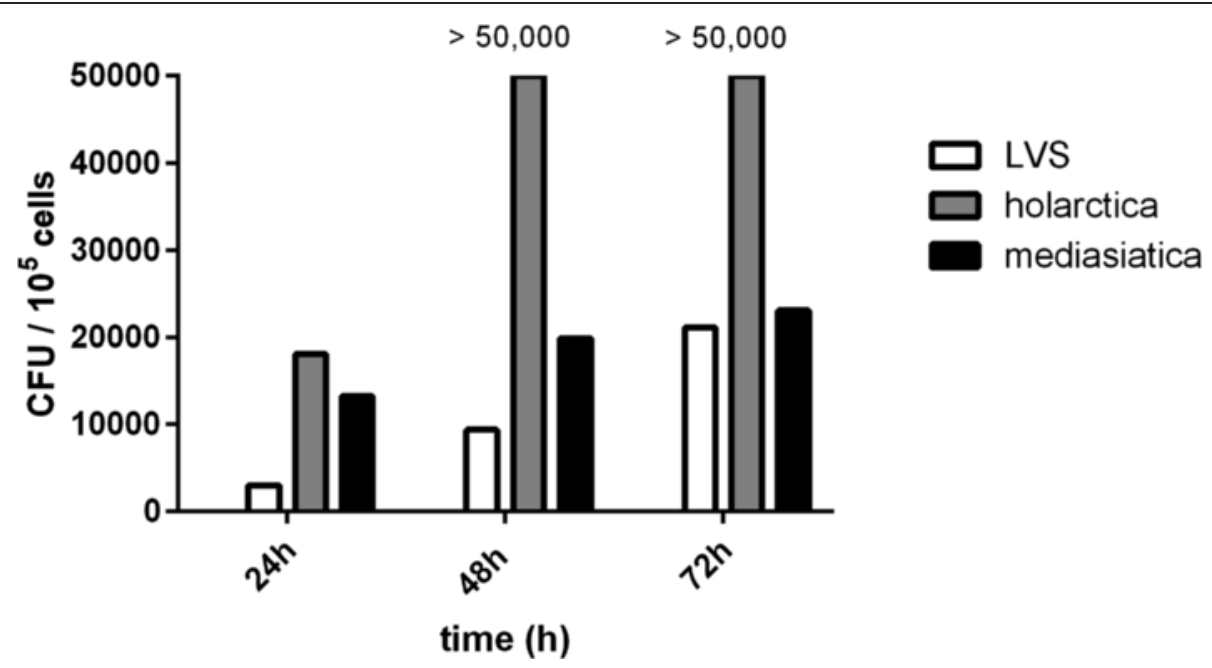

Fig. 1 Quantification of colony forming units from cell lysates of macrophage (6\%)/hepatocyte (94\%) co-cultures 24,48 and 72 h after infection with F. tularensis LVS, spp. holarctica or spp. mediasiatica plated on cysteine heart agar dishes. Data of one representative experiment out of a series of 5 independent experiments for each time point and F. tularensis strain is shown 


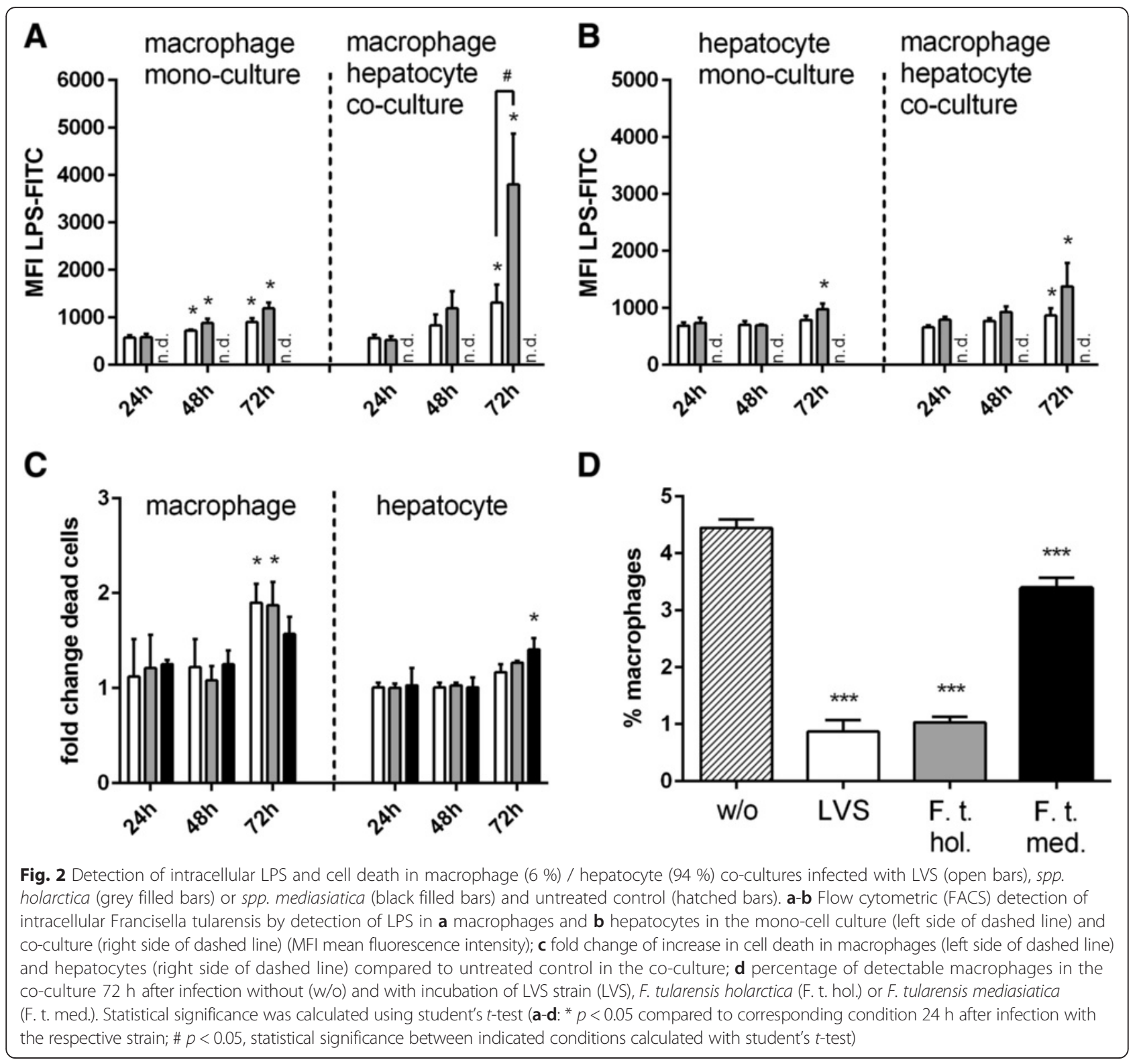

content of $6 \%$ in the co-culture (94\% hepatocytes) (Additional file 2: Figure S2A). An increase in the macrophage content in the cell cultures resulted in a diminished LPS signal reflecting first, F. tularensis preferentially enter macrophages compared to hepatocytes, and second, in consequence bacterial MOI per individual macrophage cell is reduced in the co-culture.

The strong increase in replication rate of $F$. tularensis $s s p$. holarctica in macrophages and hepatocytes in the co-culture compared with respective mono-cell cultures, indicates a supportive function of hepatocytes for bacterial replication (Fig. 2a, b). Furthermore, we observed an increased proportion of dead macrophages infected with the LVS strain and F. tularensis ssp. holarctica in the co- culture after $72 \mathrm{~h}$, whereas at the same time point $F$. tularensis ssp. mediasiatica induced a significant onset of cell death preferentially in hepatocytes, but not in macrophages (Fig. 2c). An analysis of the total percentage of macrophages revealed that infection with all three F. tularensis strains resulted in a significantly diminished macrophage numbers after $72 \mathrm{~h}$ in the co-culture. A comparative analysis with different proportions of macrophages in the co-culture revealed that a decline of present macrophages correlated with the detected LPS signal confirming bacterial infection and related subsequent cell death (Additional file 2: Figure S2B). Thus, in the subsequent experiments co-cultures of $6 \%$ macrophage and $94 \%$ hepatocytes were used to enable 
sufficient bacterial infection rate and allowing an efficient study of the related cellular response. Under these conditions the observed loss of detectable macrophages in response to bacterial infection was most prominent in cell cultures infected with LVS and $F$. tularensis ssp. holarctica compared to $F$. tularensis ssp. mediasiatica (Fig. 2d).

Induction of apoptosis in macrophages in response to infection with all three $F$. tularensis strains was detected in the co-cultures with hepatocytes at the level of cleaved caspase-3. In these assays, infection with $F$. tularensis ssp. holarctica caused the highest rate of apoptosis induction in macrophages $24 \mathrm{~h}$ and $72 \mathrm{~h}$ post infection (Fig. 3b-d, b).

It has been demonstrated in mice that after $16 \mathrm{~h}$ of intravenous infection with $F$. tularensis infectious foci in liver tissue are formed and accompanied by infiltration of both neutrophils and monocytes [20]. Infected mice developed typical signs of hepatitis within $24-72 \mathrm{~h}$ post infection [21] and during this period, pro-inflammatory cytokines, including interleukin (IL)- $1 \beta$, IL-6, and tumor necrosis factor (TNF) $\alpha$ were secreted at the active sites of infection [11, 22-24]. To characterize the inflammatory response in our humanized co-culture model the secretion of these pro-inflammatory cytokines in response to $F$. tularensis infection was measured in the supernatant of the cell culture by cytometric bead arrays (CBA). In addition, the release of fractalkine (FKN, CX3CL1), a chemokine that controls the survival of invading monocytes and their differentiation into functionally diverse macrophage subsets upon liver injury [25] was analyzed by CBA. In chronic liver injury FKN elicits liver protective functions via its receptor CX3CR1 by promoting hepatic macrophage survival and restriction of pro-inflammatory macrophage polarization [26, 27].

We observed a robust and sustained inflammatory response upon infection with all three $F$. tularensis strains up to $72 \mathrm{~h}$ after infection. TNF $\alpha$ and IL-1 $\beta$ levels were highest $24 \mathrm{~h}$ after infection and subsequently declined in the further course of culture (Fig. 4a, b). A release of IL6 was similarly triggered by infection with all Francisella strains after $24 \mathrm{~h}$. Whereas IL-6 levels declined in $F$. tularensis ssp. holarctica and ssp. mediasiatica infected co-cultures up to $72 \mathrm{~h}$, IL-6 levels remained stable upon infection with the LVS strain (Fig. 4c). In contrast to the release of pro-inflammatory cytokines TNF $\alpha, \mathrm{IL}-1 \beta$ and IL-6, the release of FKN increased from $24 \mathrm{~h}$ up to $72 \mathrm{~h}$ post infection (Fig. 4d). Interestingly, FKN release negatively correlated with pro-inflammatory cytokine release in a time-dependent as well as strain-specific manner, indicating that the release of the FKN chemokine negatively correlates with $F$. tularensis-mediated inflammation response. Among all three F. tularensis strains tested, $F$. tularensis ssp. mediasiatica infection induced the secretion of only low FKN levels but high amounts of TNF $\alpha$ and IL-1 $\beta$ were secreted relative to the LVS strain and F. tularensis ssp. holarctica.

F. tularensis attempts to evade the primary immune response by intracellular replication [3]. To study whether its presence in the cell culture is masked to suspended immune cells, we co-incubated infected cells with human white blood cells (WBC) seeded in a transwell filter above the co-cultures. In this setting we were not able to detect a significant number of migrating and adhesive leukocytes to the infected cell cultures. Only small amounts of less than $5 \%$ migrating granulocytes were detectable (Fig. 5a) in naïve and infected cell cultures, but without significant differences in respect to cell numbers (Fig. 5a). Although secretion of TNF $\alpha$ and IL-1 $\beta$ was increased, we found only minor alterations of the time- or strain-dependent IL-6- and FKN-release (Fig. 5b-e) in presence of co-incubated WBC.

\section{Discussion}

It has been suggested that hepatocytes as well as dendritic cells support the intracellular replication of $F$. tularensis without undergoing pyroptosis or apoptosis [2]. We could confirm this concept derived from the mouse models in the human cell culture model for the virulent subspecies $F$. tularensis mediasiatica but not for the subspecies holarctica or the attenuated LVS strain. In the human co-culture model we could detect a significant loss of macrophages infected with $F$. tularensis ssp. holarctica and the LVS strain, whereas the percentage of viable macrophages only slightly decreased after infection with $F$. tularensis ssp. mediasiatica.

F. tularensis is presumed to delay induction of cell death in host cells to its own advantage until exit from its intracellular environment [2]. F. tularensis ssp. mediasiatica was found to efficiently adopt this strategy by preventing macrophage cell death in order to allow efficient replication rates. It was shown that infection of the macrophage-like J774 cell line with LVS results in apoptosis [28]. However, the number of bacterial burden was not affected. In this context, a role of the caspase-3mediated cell death in favoring bacterial dissemination was assumed, but this mechanism is restricted to tissues in which macrophages play a central role in pathogen uptake and killing, such as the liver [2]. In mice, death of animals infected with $F$. tularensis appears to result from widespread sepsis and inflammation [29-31], and mortality was correlated with the extent of the inflammatory response [17] including release of pro-inflammatory IL-6, an early diagnostic marker of bacterial sepsis [32].

We observed the strongest pro-inflammatory response upon infection with $F$. tularensis ssp. mediasiatica that also induced the highest rate of apoptosis in the coculture model of all tested $F$. tularensis strains. We 


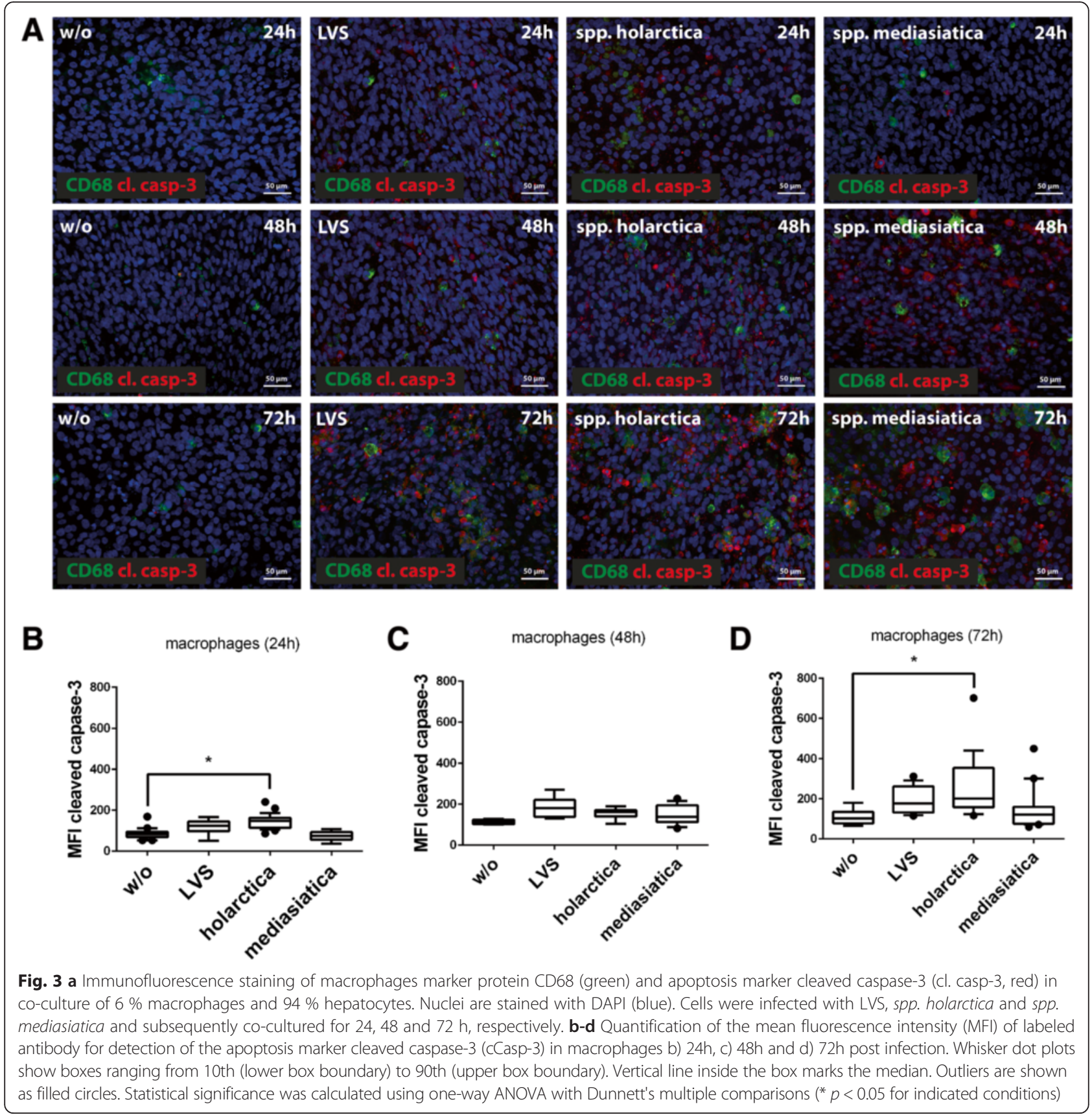

further observed a reduced level of FKN, a chemokine released by cleavage from hepatocytes upon inflammation [33] and that is able to counteract liver inflammation [26] in response to $F$. tularensis ssp. mediasiatica infection. In mice, lethal infection with F. tularensis is associated with hypercytokinemia and biochemical markers for sepsis [29-31]. This sepsis is assumed to result from delayed cytokine up-regulation and insufficient recruitment of inflammatory cells [31]. Although we observed a slight increase of TNF $\alpha$ and IL-1 $\beta$ secretion upon WBC co-incubation, no effects on IL-6 release were observed. Based on the diminished immune response of co-incubated WBC we conclude that intracellular bacteria are mainly hidden from WBC, which impedes an efficient bacterial clearance.

On one hand, the observed high rate of apoptosis induction in macrophages infected with $F$. tularensis provides the host with the advantage of early elimination of infected cells and removal of microbial replication niches. On the other hand, the extensive cell death of tissue macrophages, which is a hallmark of virulent $F$. tularensis infection [2], and the reduced recruitment of 


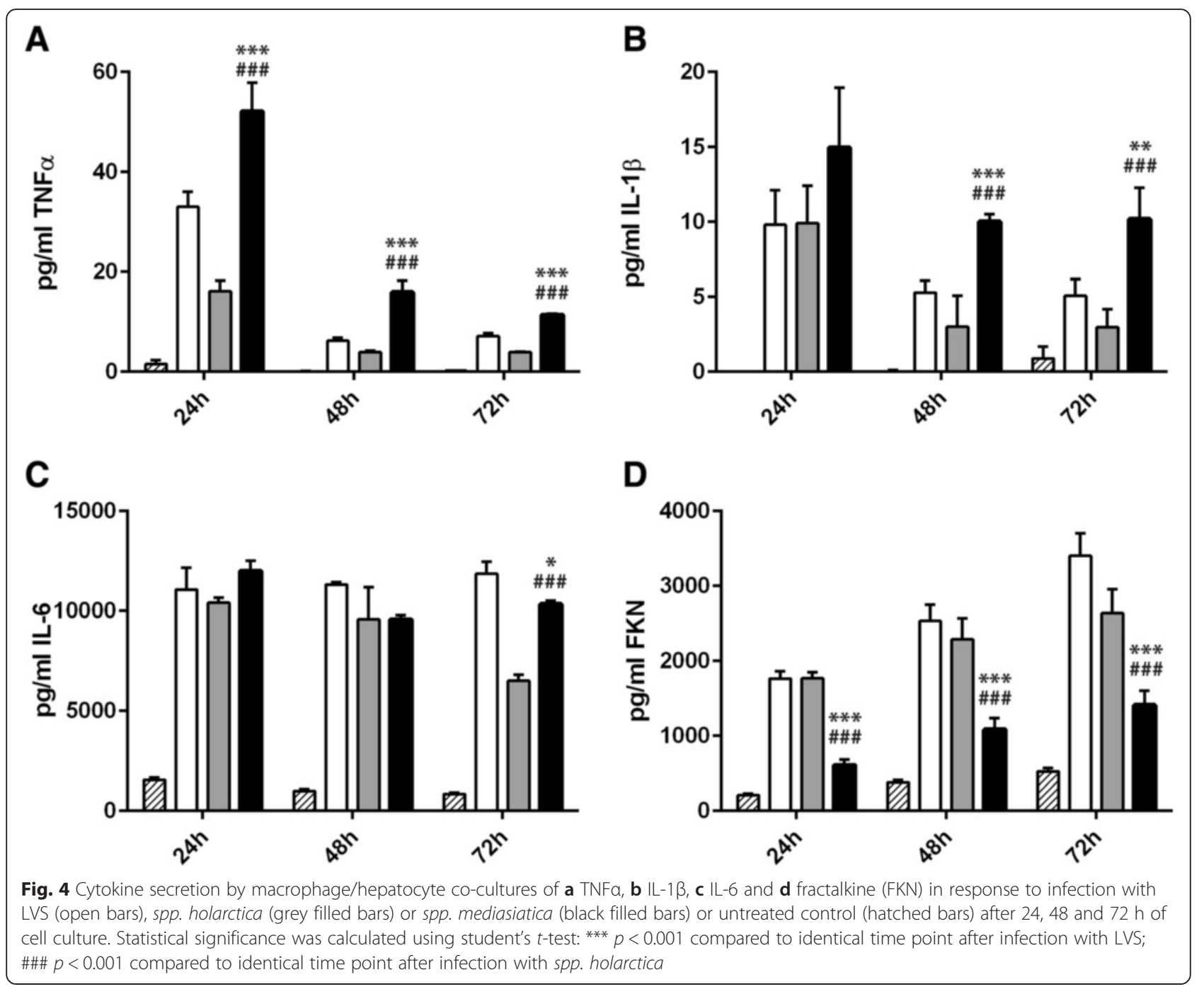

phagocytes to the sites of infection favors the dissemination of bacteria and diminishes important innate immune responses [2, 29]. This double-edged sword needs to be tightly regulated by the host to ensure an efficient host defense against $F$. tularensis infection.

\section{Conclusions}

We could demonstrate that $F$. tularensis infection and related cellular immune response can be investigated in a human cell co-culture model of monocyte-derived macrophages and hepatocytes. This co-culture model depicts strain-specific virulence and the associated pathogenic potential of the tested F. tularensis strains. Using this model we could demonstrate that $F$. tularensis strains efficiently hide and replicate in macrophages depending on their virulent potential and thereby avoid detection by co-incubated WBC and subsequent aggravated immune response. We believe that the cell coculture approach presented herein is a valuable tool for further, more detailed in vitro studies of $F$. tularensis pathophysiology. The potential of this co-culture models to study other intracellular persisting pathogens affecting the human liver will be characterized in further studies.

\section{Methods}

Cell isolation and culture

\section{HepaRG hepatocytes}

HepaRG cells were obtained from Biopredic International (Rennes, France). Undifferentiated HepaRG were seeded at a density of $2,7 \times 10^{4}$ cells $/ \mathrm{cm}^{2}$ per well of a 12 well-plate and cultured in William's Medium E (Biochrom, Berlin, Germany) containing $10 \%$ (v/v) FCS (GIBCO, Darmstadt, Germany), $5 \mu \mathrm{g} / \mathrm{ml}$ insulin (Sigma-Aldrich, Steinheim, Germany), $2 \mathrm{mM}$ glutamine (GIBCO), $5 \times 10^{-5} \mathrm{M}$ hydrocortisone-hemisuccinate (Sigma-Aldrich) and $100 \mathrm{U} / \mathrm{ml}$ penicillin $/ 100 \mu \mathrm{g} / \mathrm{ml}$ streptomycin mixture (Pen/Strep) (GIBCO). The cells were cultured in a humidified cell incubator at $5 \% \mathrm{CO}_{2}$ and $37{ }^{\circ} \mathrm{C}$ for 


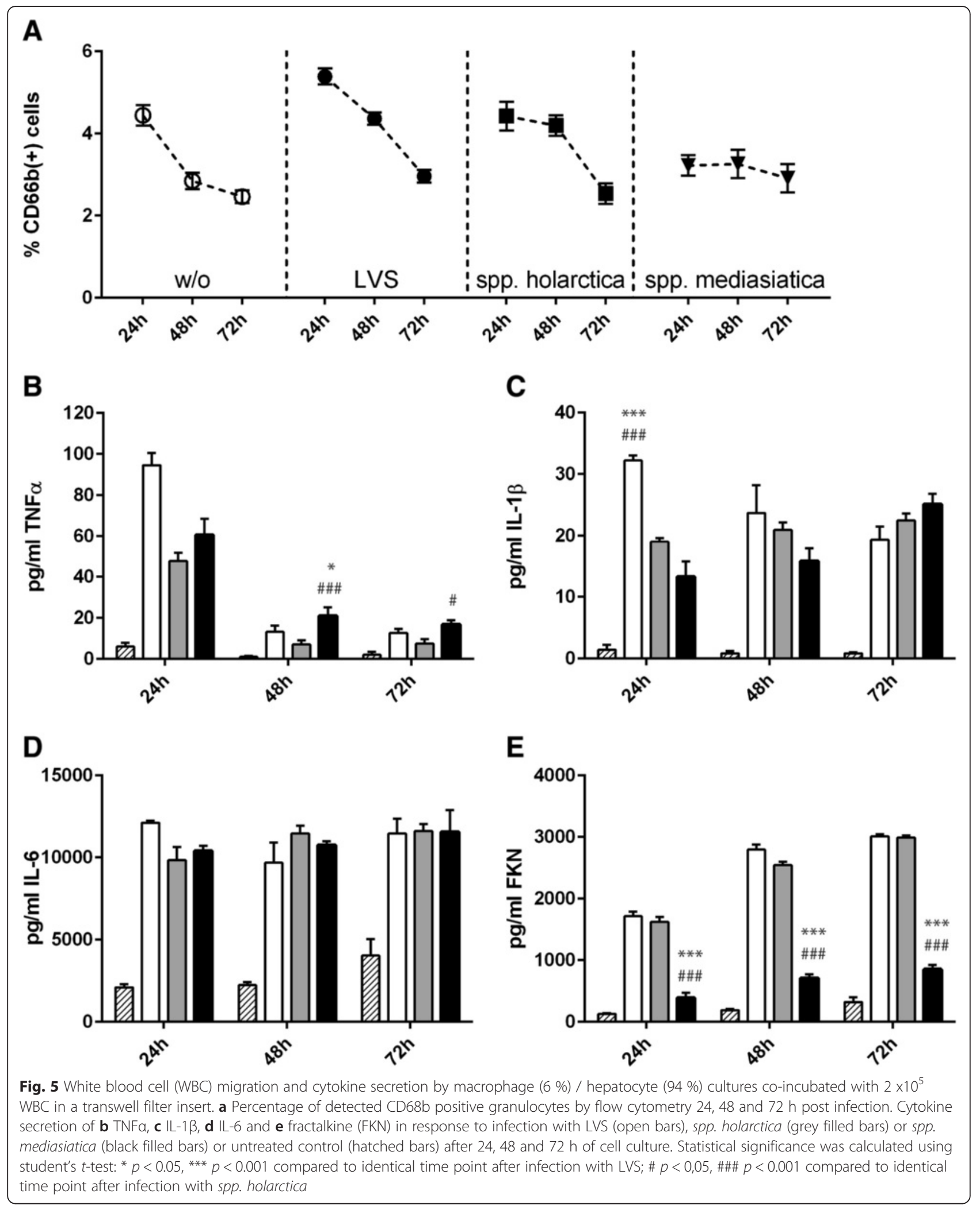


14 days before differentiation. Medium was renewed every 3-4 days. Cell differentiation was induced for 14 days in the presence of $2 \%(\mathrm{v} / \mathrm{v})$ DMSO (SigmaAldrich) as described [34]. At day 8 before starting the infection with bacteria. Primary macrophages: PBMC were isolated from whole blood which was collected from healthy human donors that were informed about the aim of the study and gave written informed consent. The study was approved by the ethics committee of the Jena University Hospital. PBMCs were isolated by Ficoll density gradient centrifugation as described previously $[35,36]$ and monocytes isolated from PBMCs with the Dynabeads ${ }^{-C D 14}$ Isolation Kit according to the manufacturer's protocol (Life Technologies, Darmstadt, Germany). For macrophages differentiation monocytes were seeded into each well of 12 well plates and cultured for 6 days in X-VIVO 15 medium (Lonza, Cologne, Germany) supplemented with $10 \%$ (v/v) autologous human serum, $10 \mathrm{ng} / \mathrm{ml}$ human granulocyte macrophage colony-stimulating factor (GM-CSF) (PeproTech, Hamburg, Germany) without antibiotics. Medium was exchanged after three days. For co-culture of macrophages and hepatocytes, monocytes were plated and differentiated for 6 days to macrophages. HepaRG cells were subsequently added to the macrophage culture. Total percentage of macrophages in the co-culture was controlled by seeding different monocyte cell densities and filling up with hepatocytes to a resulting total cell density of $1.0 \times 10^{5} \mathrm{cell} / \mathrm{cm}^{2}$ used for all tested cocultures.

\section{White blood cell isolation}

WBCs were isolated from $9 \mathrm{ml}$ EDTA-blood using the erythrocyte lysis buffer of the QIAamp RNA Blood Mini Kit (Qiagen) followed by three washing steps in PBS containing 2 mM EDTA.

\section{Francisella tularensis culture}

Francisella tularensis ssp. holarctica (FLI isolate $06 \mathrm{~T}$ 0001 and live vaccine strain-LVS) and ssp. mediasiatica (F63) were provided by the cryobank of the German National Reference Laboratory for Tularemia, plated and cultured on cysteine heart agar (CHA) in a humidified incubator at 4,5\% $\mathrm{CO}_{2}$ and $37{ }^{\circ} \mathrm{C}$ for $72 \mathrm{~h}$. Subsequently, colonies were suspended in PBS.

\section{Infection}

At the day of infection, bacterial suspensions were adjusted to an $\mathrm{OD}_{600}=0.4$, corresponding to $5 \times 10^{9}$ bacteria/ml. Cell cultures were incubated with $F$. tularensis bacteria (MOI 100:1) for $2 \mathrm{~h}$ in a humidified cell incubator at $4,5 \% \mathrm{CO}_{2}$ and $37{ }^{\circ} \mathrm{C}$. Subsequently, the bacterial suspension was removed, washed with Williams medium $\mathrm{E}$ and further incubated in William's Medium E containing $10 \%(\mathrm{v} / \mathrm{v})$ FCS, $5 \mu \mathrm{g} / \mathrm{ml}$ insulin, $2 \mathrm{mM}$ glutamine, $5 \times 10^{-5} \mathrm{M}$ hydrocortisone-hemisuccinate and $10 \mu \mathrm{g} / \mathrm{ml}$ gentamicin (Sigma-Aldrich, Taufkirchen, Germany) for $30 \mathrm{~min}$ to kill extracellular bacteria. Infected cells with persisting Francisellae were than washed with PBS and cultured for indicated times in William's Medium E containing $10 \%$ (v/v) FCS, $5 \mu \mathrm{g} / \mathrm{ml}$ insulin, $2 \mathrm{mM}$ glutamine, $5 \times 10^{-5} \mathrm{M}$ hydrocortisonehemisuccinate without antibiotics.

\section{White blood cell co-incubation}

$2 \times 10^{5}$ freshly isolated white blood cells were transferred into each cell culture inserts with $8 \mu \mathrm{m}$ pores (Merck Millipore, Darmstadt) hanging above F.tularensis pre-inoculated co-cultures of HepaRG/macrophages. WBCs were incubated for up to $72 \mathrm{~h}$ with the infected co-cultures. Subsequently, inserts were discarded and cells in the subjacent well were harvested for flow cytometry. Granulocytes and T-cells were stained with fluorochrome-conjugated antibodies against CD66b and CD3 (BD Biosciences) and analyzed by flow cytometry.

\section{Immunofluorescence staining}

Cells were fixed with $4 \%$ paraformaldehyde for $10 \mathrm{~min}$ at room temperature (RT). Staining was done with antibodies against CD68 (BD Biosciences, Heidelberg, Germany), cleaved caspase 3 (cCasp-3) (Cell Signaling Technology, Leiden, Netherlands), and goat-anti-rabbitCy3 (Dianova, Hamburg, Germany) and goat-antimouse-AlexaFluor488 (AF488) as secondary antibodies, and DAPI (Life Technologies, Karlruhe, Germany). Samples were embedded into fluorescent mounting medium (Dako, Hamburg, Germany). Subsequently, imaging was performed on an AXIO Observer Z1 fluorescence microscope equipped with Apotome 2 (Carl Zeiss AG, Jena, Germany).

\section{Image analysis}

Analysis of cleaved caspase-3 fluorescence signals in macrophages was done with ImageJ2 software. Specifically, macrophages were detected based on the expression of the cell-type-specific marker protein CD68 stained with AF488 coupled secondary antibody and cell borders of the cell marked as region of interest (ROI). ROI's were than analyzed for fluorescence signals of cCasp-3 and mean fluorescence intensity (MFI) was calculated and plotted in a whisker box plot diagram.

\section{Colony forming unit assay}

After indicated incubation times, cells were lysed in $1 \mathrm{ml}$ Aqua bidest. for $10 \mathrm{~min}$ at RT. Subsequently, 20-100 $\mu \mathrm{l}$ of the lysate were transferred and streaked on cysteine heart agar without antibiotics. Agar plates were 
cultivated for 2 days in a humidified incubator at 4,5\% $\mathrm{CO}_{2}$ and $37{ }^{\circ} \mathrm{C}$. Afterwards colonies were counted.

\section{Live/dead staining}

Macrophages and hepatocytes were detached from cell culture dishes using $4 \mathrm{mg} / \mathrm{ml}$ Lidocaine and $5 \mathrm{mM}$ EDTA (Sigma Aldrich) in PBS (Lonza, Cologne, Germany), centrifuged at $300 \mathrm{x} g$ at room temperature (RT) for 6 min, washed two times with PBS at RT and stained with LIVE/DEAD ${ }^{\circ}$ Cell Viability assay (Life Technologies) for $30 \mathrm{~min}$ according to the manufacturer's instructions.

\section{FACS analysis}

Macrophages were stained with antibody against CD45APC-Cy7 (BD Bioscience, Heidelberg, Germany) and fixed with Inside Stain kit (Miltenyi Biotec, Bergisch Gladbach) according to manufacturer's recommendations for permeabilization of cells thus allowing detection of F. tularensis LPS. Intracellular Francisellae were stained with $2,5 \mu \mathrm{g} / \mathrm{ml}$ FITC-conjugated antibody versus F. tularensis LPS (clone FB11 - HyTEST Ltd., Turku, Finland). Clone FB 11 does not recognize LPS of $F$. tularensis ssp. mediasiatica. Flow cytometry was performed on a BD FACS-Canto II (BD Biosciences) with FACSDiva software and analyzed using FlowJo X software (FlowJo LLC, Ashland, OR, USA).

\section{Cytometric bead array (CBA)}

Supernatants were collected after indicated time periods and immediately frozen at $-80{ }^{\circ} \mathrm{C}$. Cytokines were detected using CBA assay (BD Biosciences) according to the manufacturer's protocol. Secretion of TNF $\alpha$, IL-1 $\beta$, IL-6, fractalkine and IFN $\alpha$ was analyzed using standard CBA flex sets. Analysis was performed on a BD FACSCanto II cytometer with FACSDiva software. Data analysis was performed using FCAP Array V3 software (Softflow, Pecs, Hungary).

\section{Statistics}

All results are represented as mean of the performed experiments with standard deviation. Statistic tests were done with two-tailed, non-paired Student's $t$-test or oneway ANOVA with Dunnett's multiple comparisons test between indicated conditions. Statistical analysis was performed using GraphPad Prism 6.07 software (Graphpad Software, La Jolla, CA, USA). For CFU analysis data of one representative experiment out of a series of 5 independent experiments for each time point and $\mathrm{F}$. tularensis strain is shown in Fig. 1 and Additional file 1: Figure S1.

\section{Additional files}

Additional file 1: Figure S1. Colony forming units assay from cel lysates of macrophage (6\%)/hepatocyte (94\%) co-cultures 24,48 and $72 \mathrm{~h}$ after infection with $\mathrm{F}$. tularensis LVS, spp. holarctica or spp. mediasiatica plated on cysteine heart agar dishes. Data of one representative experiment out of a series of 5 independent experiments for each time point and F. tularensis strain is shown. (TIF $77516 \mathrm{~kb}$ )

Additional file 2: Figure S2. Detection of intracellular LPS in macrophage / hepatocyte co-cultures infected with LVS (open bars), spp. holarctica (grey filled bars) or spp. mediasiatica (black filled bars) and untreated control (hatched bars). A) Different amounts of macrophages in the co-culture were tested $(6,12$ and $22 \%$ of macrophages on total cell count). Flow cytometric detection of intracellular LPS in macrophages (MFI mean fluorescence intensity); B-D) percentage of remaining detectable macrophages after infection of the co-cultures with B) $6 \%$ macrophages/ $94 \%$ hepatocytes, C) $12 \%$ macrophages/ $88 \%$ hepatocytes and D) $22 \%$ macrophages/ $88 \%$ hepatocytes $72 \mathrm{~h}$ post infection. (TIF $32735 \mathrm{~kb}$ )

\section{Abbreviations}

CHA: Cysteine heart agar; CFU: Colony forming unit; CX3CL1: Fractalkine; DMEM: Dulbecco's modified eagle medium; ECM: Endothelial cell medium; FKN: Fractalkine; F. tularensis: Francisella tularensis; GM-CSF: Granulocytemacrophage colony stimulating factor; IL: Interleukin;

LPS: Lipopolysaccharide; LVS: Live vaccine strain; MOI: Multiplicity of infection; Pen/Strp.: Penicillin/Streptomycin; RT: Room temperature; spp.: Subspecies; WBC: White blood cell; v/v: Volume/Volume.

\section{Competing interests}

The authors declare that they have no competing interests.

\section{Authors' contributions}

KR performed the experiments. PO, HF, HT contributed reagents/materials/ analysis tools. KR, PO, ASM analyzed the data. OH, HT, ASM wrote the manuscript. HT, ASM designed the study and supervised experiments. All authors read and approved the final version of the manuscript.

\section{Acknowledgements}

We are grateful to the excellent technical work of Maria Franke and Margot Voigt. The authors would further like to acknowledge support of this work by grant of the German Research Platform for Zoonoses (FK 01KI1109).

\section{Author details}

${ }^{1}$ Institute of Biochemistry II, Jena University Hospital, 07743 Jena, Germany. ${ }^{2}$ Institute of Bacterial Infections and Zoonoses (IBIZ),

Friedrich-Loeffler-Institute, Federal Research Institute for Animal Health, 07743 Jena, Germany. ${ }^{3}$ Molecular Hemostaseology, Jena University Hospital, 07743 Jena, Germany. ${ }^{4}$ Center for Sepsis Control and Care, Jena University Hospital, Jena 07747, Germany.

Received: 27 August 2015 Accepted: 29 December 2015

Published online: 06 January 2016

\section{References}

1. McLendon MK, Apicella MA, Allen LA. Francisella tularensis: taxonomy, genetics, and Immunopathogenesis of a potential agent of biowarfare. Annu Rev Microbiol. 2006;60:167-85.

2. Parmely MJ, Fischer $\mathrm{J}$, Pinson DM. Programmed cell death and the pathogenesis of tissue injury induced by type A Francisella tularensis. FEMS Microbiol Lett. 2009:301:1-11.

3. del Barrio L, Sahoo M, Lantier L, Reynolds JM, Ceballos-Olvera I, Re F. Production of Anti-LPS IgM by B1a B Cells Depends on IL-1 $\beta$ and Is Protective against Lung Infection with Francisella tularensis LVS. PLoS Pathog. 2015;11:1-20.

4. Snoy PJ. Establishing efficacy of human products using animals: the US food and drug administration's "animal rule". Vet Pathol. 2010;47(5):774-8.

5. Råberg L, Sim D, Read AF. Disentangling genetic variation for resistance and tolerance to infectious diseases in animals. Science. 2007;318:812-4. 
6. Schneider DS, Ayres JS. Two ways to survive infection: what resistance and tolerance can teach us about treating infectious diseases. Nat Rev Immunol. 2008;8:889-95.

7. Seok J, Warren HS, Cuenca AG, Mindrinos MN, Baker HV, Xu W, et al. Genomic responses in mouse models poorly mimic human inflammatory diseases. Proc Natl Acad Sci U S A. 2013;110:3507-12.

8. Keim P, Johansson A, Wagner DM. Molecular epidemiology, evolution, and ecology of Francisella. Ann N Y Acad Sci. 2007;1105:30-66.

9. De Pascalis R, Chou AY, Ryden P, Kennett NJ, Sjostedt A, Elkins KL. Models derived from in vitro analyses of spleen, liver, and lung leukocyte functions predict vaccine efficacy against the Francisella tularensis Live Vaccine Strain (LVS). MBio. 2014;5:e00936-00913-3.

10. Anthony LS, Kongshavn PA. Experimental murine tularemia caused by Francisella tularensis, live vaccine strain: a model of acquired cellular resistance. Microb Pathog. 1987;2(1):3-14.

11. Bokhari SM, Kim KJ, Pinson DM, Slusser J, Yeh HW, Parmely MJ. NK cells and gamma interferon coordinate the formation and function of hepatic granulomas in mice infected with the Francisella tularensis live vaccine strain. Infect Immun. 2008;76(4):1379-89.

12. Asare R, Abu Kwaik Y. Molecular complexity orchestrates modulation of phagosome biogenesis and escape to the cytosol of macrophages by Francisella tularensis. Environ Microbiol. 2010;12(9):2559-86.

13. Sjostedt A. Intracellular survival mechanisms of Francisella tularensis, a stealth pathogen. Microbes Infect. 2006;8(2):561-7.

14. Clemens DL, Lee BY, Horwitz MA. Virulent and avirulent strains of Francisella tularensis prevent acidification and maturation of their phagosomes and escape into the cytoplasm in human macrophages. Infect Immun. 2004;72(6):3204-17.

15. Bouwens L, Baekeland M, De Zanger R, Wisse E. Quantitation, tissue distribution and proliferation kinetics of Kupffer cells in normal rat liver. Hepatology. 1986;6(4):718-22.

16. Ishibashi H, Nakamura M, Komori A, Migita K, Shimoda S. Liver architecture, cell function, and disease. In: Semin Immunopathol. vol. 31. Berlin: Springer; 2009. p. 399-409.

17. Chiavolini D, Alroy J, King CA, Jorth P, Weir S, Madico G, et al. Identification of immunologic and pathologic parameters of death versus survival in respiratory tularemia. Infect Immun. 2008;76:486-96.

18. Guillouzo A, Guguen-Guillouzo C. Evolving concepts in liver tissue modeling and implications for in vitro toxicology. Expert Opin Drug Metab Toxicol. 2008:4(10):1279-94.

19. Hajjar AM, Harvey MD, Shaffer SA, Goodlett DR, Sjöstedt A, Edebro H, et al. Lack of in vitro and in vivo recognition of Francisella tularensis subspecies lipopolysaccharide by Toll-like receptors. Infect Immun. 2006;74:6730-8.

20. Conlan JW, North RJ. Early pathogenesis of infection in the liver with the facultative intracellular bacteria Listeria monocytogenes, Francisella tularensis, and Salmonella typhimurium involves lysis of infected hepatocytes by leukocytes. Infect Immun. 1992;60(12):5164-71.

21. White JD, Rooney JR, Prickett PA, Derrenbacher EB, Beard CW, Griffith WR. Pathogenesis of experimental respiratory tularemia in monkeys. J Infect Dis. 1964;114:277-83.

22. Cole LE, Elkins KL, Michalek SM, Qureshi N, Eaton LJ, Rallabhandi $P$, et al. Immunologic consequences of Francisella tularensis live vaccine strain infection: role of the innate immune response in infection and immunity. J Immunol. 2006;176:6888-99.

23. Wickstrum JR, Bokhari SM, Fischer JL, Pinson DM, Yeh HW, Horvat RT, et al. Francisella tularensis induces extensive caspase-3 activation and apoptotic cell death in the tissues of infected mice. Infect Immun. 2009:77:4827-36.

24. Golovliov I, Sandstrom G, Ericsson M, Sjostedt A, Tarnvik A. Cytokine expression in the liver during the early phase of murine tularemia. Infect Immun. 1995;63(2):534-8.

25. Zimmermann HW, Trautwein C, Tacke F. Functional role of monocytes and macrophages for the inflammatory response in acute liver injury. Front Physiol. 2012;3:56.

26. Aoyama T, Inokuchi S, Brenner DA, Seki E. CX3CL1-CX3CR1 interaction prevents carbon tetrachloride-induced liver inflammation and fibrosis in mice. Hepatology. 2010;52(4):1390-400

27. Karlmark KR, Zimmermann HW, Roderburg C, Gassler N, Wasmuth HE, Luedde $T$, et al. The fractalkine receptor CX(3)CR1 protects against liver fibrosis by controlling differentiation and survival of infiltrating hepatic monocytes. Hepatology. 2010;52(5):1769-82
28. Lai XH, Golovliov I, Sjostedt A. Francisella tularensis induces cytopathogenicity and apoptosis in murine macrophages via a mechanism that requires intracellular bacterial multiplication. Infect Immun. 2001;69(7):4691-4.

29. Sharma J, Mares CA, Li Q, Morris EG, Teale JM. Features of sepsis caused by pulmonary infection with Francisella tularensis Type A strain. Microb Pathog. 2011:51(1-2):39-47.

30. Sharma J, Li Q, Mishra BB, Pena C, Teale JM. Lethal pulmonary infection with Francisella novicida is associated with severe sepsis. J Leukoc Biol. 2009; 86(3):491-504.

31. Mares CA, Ojeda SS, Morris EG, Li Q, Teale JM. Initial delay in the immune response to Francisella tularensis is followed by hypercytokinemia characteristic of severe sepsis and correlating with upregulation and release of damage-associated molecular patterns. Infect Immun. 2008:76(7):3001-10.

32. Lin S, Huang Z, Wang M, Weng Z, Zeng D, Zhang Y, et al. Interleukin-6 as an early diagnostic marker for bacterial sepsis in patients with liver cirrhosis. J Crit Care. 2015;30(4):732-8.

33. Turner SL, Mangnall D, Bird NC, Blair-Zajdel ME, Bunning RA. Effects of proinflammatory cytokines on the production of soluble fractalkine and ADAM17 by HepG2 cells. J Gastrointestin Liver Dis. 2010;19(3):265-71.

34. Gripon P, Rumin S, Urban S, Le Seyec J, Glaise D, Cannie I, et al. Infection of a human hepatoma cell line by hepatitis B virus. Proc Natl Acad Sci U S A. 2002;99(24):15655-60.

35. Mosig S, Rennert K, Krause S, Kzhyshkowska J, Neunubel K, Heller R, et al. Different functions of monocyte subsets in familial hypercholesterolemia: potential function of CD14+ CD16+ monocytes in detoxification of oxidized LDL. FASEB J. 2009:23(3):866-74.

36. Wallert M, Mosig S, Rennert K, Funke H, Ristow M, Pellegrino RM, et al. Long-chain metabolites of alpha-tocopherol occur in human serum and inhibit macrophage foam cell formation in vitro. Free Radic Biol Med. 2014:68:43-51.

\section{Submit your next manuscript to BioMed Central and we will help you at every step:}

- We accept pre-submission inquiries

- Our selector tool helps you to find the most relevant journal

- We provide round the clock customer support

- Convenient online submission

- Thorough peer review

- Inclusion in PubMed and all major indexing services

- Maximum visibility for your research

Submit your manuscript at www.biomedcentral.com/submit 